\title{
TRACE-CLASS NORM MULTIPLIERS
}

\author{
ROSHDI KHALIL ${ }^{1}$
}

\begin{abstract}
In this paper we study the pointwise multipliers of the space $L^{2}(X, \nu) \hat{\otimes}$ $L^{2}(Y, \mu)$ for different measure spaces $(X, \nu)$ and $(Y, \mu)$. First we consider the case where $X$ and $Y$ are finite sets, then we use this to study the multipliers of $L^{2}(I, v) \hat{\otimes} L^{2}(I, v)$, where $I$ is the unit interval and $v$ any Borel measure on $I$.
\end{abstract}

0. Introduction. Let $A$ be a Banach space of complex-valued functions on a measure space $(X, v)$ such that $A$ vanishes at no point of $X$. A multiplier of $A$ is a complex-valued function $\varphi$ on $X$ such that $\varphi f \in A$ for all $f \in A$, where $(\varphi f)(x)=$ $\varphi(x) f(x)$. Clearly, $M(A)$, the set of all multipliers of $A$, forms an algebra which will be called the multiplier algebra of $A$.

Multiplier algebras have been studied by Schur, [8], for the space of matrices describing bounded linear operators on $l^{2}$, and by Bennett, [2], for certain other spaces of matrices. Bennett proved the result: a matrix $F: l^{p} \rightarrow l^{q}$ is a multiplier of $L\left(l^{p}, l^{q}\right)$ (the space of bounded linear operators from $l^{p}$ into $l^{q}$ ) if and only if, for each $d \in l^{p}$, the matrix $F \circ(\operatorname{diag} d): l^{p^{*}} \rightarrow l^{\infty}$ is $(q, q)$-absolutely summing. The space $L^{2}(X, \nu) \hat{\otimes} L^{2}(X, v)$, the completion of the projective tensor product with itself [1], can be realized as a space of functions on $(X \times X, \nu \otimes \nu)$. The author [5] showed that $\pi\left(l^{2} \hat{\otimes} l^{2}\right)$ is isomorphic to the space of all functions $\varphi$ on $N \times N$ ( $N$ is the set of natural numbers) such that there exists a sequence $\left(\varphi_{n}\right)_{n-1}^{\infty}$ uniformly bounded in $l^{\infty} \hat{\otimes} l^{\infty}$ which converges to $\varphi$ pointwise. The previous result of the author is included implicitly in that of Bennett [2].

The object of this paper is to study the multiplier algebra of $L^{2}(X, \nu) \hat{\otimes} L^{2}(X, \nu)$ for a nondiscrete space $X$. In particular we study the multiplier algebra of $L^{2}(I, \nu) \hat{\otimes} L^{2}(I, \nu)$, where $I$ is the unit interval and $\nu$ is any Borel measure on $I$. As is known [7] $L^{2}(X, \nu) \hat{\otimes} L^{2}(X, \nu)$ is isometrically isomorphic to the space of trace-class operators on $L^{2}(X, \nu)$. This implies that if $\varphi \in \Re\left(L^{2}(I, \nu) \hat{\otimes} L^{2}(I, \nu)\right)$,

$$
\|\varphi\|_{M}=\sup |\langle\varphi \cdot u \otimes v, U\rangle|
$$

where the supremum is taken over all atoms $u \otimes v$ and all operators $U$ in the unit balls of $L^{2}(X, \nu) \hat{\otimes} L^{2}(X, \nu)$ and $L\left(L^{2}(X, \nu)\right)$ respectively.

Throughout this paper, $H$ denotes a real (or complex) Hilbert space of dimension $n$. If $A$ is a Banach space then $A^{*}$ denotes its dual and $L(A)$ is the space of bounded linear operators on $A$. An atom in $A \hat{\otimes} A$ is an element of the form

Received by the editors October 17, 1978 and, in revised form, March 20, 1979.

AMS (MOS) subject classifications (1970). Primary 46L20; Secondary 47C10.

${ }^{1}$ Supported in part by an FCAC Grant from the government of Québec. This is based on part of the author's Ph.D thesis, McGill University, under Professor S. Drury. The author is grateful for Professor Drury's assistance. 
$f \otimes g, f$ and $g$ are in $A$. For $a \in A,\|a\|_{A}$ denotes the norm of $a$ in $A$. The space of $p$-summable sequences will be denoted by $l^{p}$, and $l^{\infty}$ stands for the space of bounded sequences. Finally, $l_{n}^{2}$ will denote the $n$-dimensional space of square summable sequences, $Z_{n}^{+}$stands for the set $\{1,2, \ldots, n\}$, and $p^{*}$ is the conjugate of $p\left(1 / p+1 / p^{*}=1\right)$.

1. Multipliers and normal contractions. We denote by $H$ a complex Hilbert space. The set $D=\{Z \in C|| Z \mid<1\}$ will denote the unit disc, and $C(D)$ the space of continuous functions on $D$. For any pair of normal contractions $S$ and $T$ on $H$ and for any element $\varphi$ in $C(D) \hat{\otimes} C(D)$, one can make sense of $\varphi(S, T)$. Let $E_{1}=$ $\left\{\lambda_{1}, \ldots, \lambda_{n}\right\}$ and $E_{2}=\left\{n_{1}, \ldots, n_{n}\right\}$ be any two sets in $D$, where $n$ is the dimension of $H$. If $Z_{n}^{+}$is the set $\{1, \ldots, n\}$, then consider the function $\psi: Z_{n}^{+} \times Z_{n}^{+} \rightarrow$ C defined by

$$
\psi(i, j)=\varphi\left(\lambda_{i}, \eta_{j}\right), \quad 1<i, j<n .
$$

Then we prove the following.

THEOREM 1.1. The following are equivalent.

(i) $\psi \in M\left(l_{n}^{2} \hat{\otimes} l_{n}^{2}\right)$ and $\|\psi\|_{M}<1$.

(ii) $\|\varphi(S, T)\|_{L(H)}<1$ for any pair of normal (not necessarily commuting) contractions $S$ and $T$ on $H$ whose eigenvalues are the elements of the sets $E_{1}$ and $E_{2}$ respectively.

Proof. (i) $\rightarrow$ (ii). Let $S$ and $T$ be any pair of normal contractions on $H$ with $E_{1}$ and $E_{2}$ their sets of eigenvalues. Let $\left\{e_{1}, \ldots, e_{n}\right\}$ and $\left\{f_{1}, \ldots, f_{n}\right\}$ be two sets of orthonormal vectors such that

$$
S e_{j}=\lambda_{i} e_{i} \text { and } T f_{j}=\eta_{j} f_{j}, \quad 1<i, j<n .
$$

Let $\sum_{k=1}^{\infty} c_{k} g_{k} \otimes h_{k}$ be a representation of $\varphi$ in $C(D) \hat{\otimes} C(D)$, and let $e$ and $f$ be any two unit vectors in $H$. If $e=\sum_{i=1}^{n} a_{i} e_{i}$ and $f=\sum_{j=1}^{n} b_{j} f_{j}$, where $a=\left(a_{1}, \ldots, a_{n}\right)$ and $b=\left(b_{1}, \ldots, b_{n}\right)$ are unit vectors in $l_{n}^{2}$, then we have

$$
\begin{aligned}
\langle\varphi(S, T) f, e\rangle \mid & =\left|\left\langle\sum_{k=1}^{\infty} c_{k} g_{k}(S) h_{k}(T) f, e\right\rangle\right| \\
& =\left|\sum_{i, j=1}^{n} \varphi\left(\lambda_{i}, \eta_{j}\right) \bar{a}_{i} b_{j}\left\langle f_{j}, e_{i}\right\rangle\right| \\
& =\left|\sum_{i j=1}^{n} \psi(i, j) u(i) v(j) U(j, i)\right|,
\end{aligned}
$$

where $u(i)=\bar{a}_{i}, v(j)=b_{j}, U(j, i)=\left\langle f_{j}, e_{i}\right\rangle$. Hence $u \otimes v$ is an atom in $l_{n}^{2} \hat{\otimes} l_{n}^{2}$ and $U$ is a unitary operator on $l_{n}^{2}$. Assumption (i) then implies that $|\langle\varphi(S, T) f, e\rangle|$ $<1$. This proves (ii).

Conversely (ii) $\rightarrow$ (i). Let $u \otimes v$ be any atom of unit norm in $l_{n}^{2} \hat{\otimes} l_{n}^{2}$ and $U$ be any unitary operator on $l_{n}^{2}$. If $\left\{e_{1}, \ldots, e_{n}\right\}$ and $\left\{f_{1}, \ldots, f_{n}\right\}$ are two sets of orthonormal vectors in $H$ such that $U(i, j)=\left\langle f_{i}, e_{j}\right\rangle$, then define two linear operators $S$ and $T$ on $H$ as follows. 


$$
S\left(\sum_{i=1}^{n} a_{i} e_{i}\right)=\sum_{i=1}^{n} a_{i} \bar{\lambda}_{i} e_{i}, \quad T\left(\sum_{j=1}^{n} b_{j} f_{j}\right)=\sum_{j=1}^{n} b_{j} \eta_{j} f_{j}
$$

Then $S$ and $T$ are two normal contractions on $H$ with sets of eigenvalues $E_{1}$ and $E_{2}$ respectively. Set $e=\sum_{i=1}^{n} \bar{u}(i) e_{i}, f=\sum_{j=1}^{n} v(j) f_{j}$. Then

$$
\begin{aligned}
\left|\sum_{i, j=1}^{n} \psi(i, j) u(i) v(j) U(j, i)\right| & =\left|\sum_{i, j=1}^{n} \varphi\left(\lambda_{i}, \eta_{j}\right) u(i) v(j)\left\langle f_{j}, e_{i}\right\rangle\right| \\
& =|\langle\varphi(S, T) f, e\rangle|<1
\end{aligned}
$$

by assumption (ii). Since the unit ball of $L\left(l_{n}^{2}\right)$ is just the closed convex hull of the set of unitary operators [6], (i) follows. The proof of the theorem is complete.

Since $M\left(l_{n}^{2} \hat{\otimes} l_{n}^{2}\right)=l_{n}^{\infty} \hat{\otimes} l_{n}^{\infty},[5]$, up to norm equivalence, it follows that if $\varphi$ is a polynomial on $D \times D$ then

$$
\|\varphi\|_{C(D) \hat{\otimes} C(D)} \leqslant C \cdot\|\varphi(S, T)\|_{L(H)},
$$

for some constant $C$ independent of $\varphi$, for all pairs of normal contractions $S$ and $T$ on $H$.

2. Multipliers and spectral measures. Let $X$ be a set, $F$ a $\sigma$-algebra of subsets of $X$. A map $P, P: F \rightarrow L(H)$ is called a spectral measure on $X$ if the range of $P$ is contained in the set of projections in $L(H)$, if disjoint sets are taken to orthogonal projections, and if $P(X)=I$. Further the set function $\mu_{x}: F \rightarrow C, \mu_{x}(E)=$ $\langle P(E) x, x\rangle$ is a measure on $X$ for all $x \in H$. Now taking $X$ to be the set $Z_{n}^{+}$and $F$ to be the family of all subsets of $Z_{n}^{+}$, we prove

THEOREM 2.1. The following are equivalent.

(i) $\varphi \in M\left(l_{n}^{2} \hat{\otimes} l_{n}^{2}\right)$ and $\|\varphi\|_{M} \leqslant 1$.

(ii) $\left\|\sum_{i, j=1}^{n} \varphi(i, j) P(i) Q(j)\right\|_{L(H)} \leqslant 1$ for every pair of (not necessarily commuting) spectral measures $P, Q$ on $Z_{n}^{+}$.

Proof. (i) $\rightarrow$ (ii). Let $P$ and $Q$ be any two spectral measures on $Z_{n}^{+}$. Set $P(i) H=A_{i}$ and $Q(j) H=B_{j}, 1 \leqslant i, j \leqslant n$. Then

$$
H=A_{1} \oplus \cdots \oplus A_{n}, \quad H=B_{1} \oplus \cdots \oplus B_{n} .
$$

Let $a$ and $b$ be any two unit vectors in $H$. If $P(i) a=a_{i}$ and $Q(j) b=b_{j}$, then, agreeing not to divide by zero,

$$
\begin{aligned}
& a=\left\|a_{1}\right\| \cdot a_{1} /\left\|a_{1}\right\|+\cdots+\left\|a_{n}\right\| \cdot a_{n} /\left\|a_{n}\right\|, \\
& b=\left\|b_{1}\right\| \cdot b_{1} /\left\|b_{1}\right\|+\cdots+\left\|b_{n}\right\| \cdot b_{n} /\left\|b_{n}\right\| .
\end{aligned}
$$

Set $e_{i}=a_{i} /\left\|a_{i}\right\|$ if $a_{i} \neq 0$ and $e_{i}=0$ otherwise. Similarly $f_{j}=b_{j} /\left\|b_{j}\right\|$ if $b_{j} \neq 0$ and $f_{j}=0$ otherwise. Then $U(i, j)=\left\langle f_{i}, e_{j}\right\rangle$ is a contraction on $l_{n}^{2}$. Put $u(i)=\left\|a_{i}\right\|$ and $v(j)=\left\|b_{j}\right\|$, then $u \otimes v$ is an atom of unit norm in $l_{n}^{2} \hat{\otimes} l_{n}^{2}$. Now

$$
\begin{aligned}
\left|\sum_{i, j=1}^{n} \varphi(i, j)\langle Q(j) b, P(i) a\rangle\right| & =\left|\sum_{i, j=1}^{n} \varphi(i, j) u(i) v(j) U(j, i)\right| \\
& <\|\varphi\|_{M}<1 .
\end{aligned}
$$


This proves (ii).

Conversely (ii) $\rightarrow$ (i). Let $u \otimes v$ be any atom in the unit ball of $l_{n}^{2} \hat{\otimes} l_{n}^{2}$ and $U$ be any unitary operator in $L\left(l_{n}^{2}\right)$. Choose $\left\{e_{1}, \ldots, e_{n}\right\}$ and $\left\{f_{1}, \ldots, f_{n}\right\}$ to be two sets of orthonormal vectors in $H$ such that $U(i, j)=\left\langle f_{i}, e_{j}\right\rangle$. Put $y=\sum_{i=1}^{n} \bar{u}(i) e_{i}, x=$ $\sum_{j=1}^{n} v(j) f_{j}$, and define $P(i)=$ Projection on the span of $\left\{e_{i}\right\}$ and $Q(j)=$ Projection on the span of $\left\{f_{j}\right\}$. Then we have

$$
\begin{aligned}
\left|\sum_{i, j=1}^{n} \varphi(i, j) u(i) v(j) U(j, i)\right| & =\left|\sum_{i, j=1}^{n} \varphi(i, j)\langle Q(j) x, P(i) y\rangle\right| \\
& <\left\|\sum_{i, j=1}^{n} \varphi(i, j) P(i) Q(j)\right\|_{L(H)}<1 .
\end{aligned}
$$

This completes the proof of the theorem.

Theorem 2.1 can be restated as follows.

THEOREM 2.1'. The following are equivalent.

(i) $\varphi \in M\left(l_{n}^{2} \hat{\otimes} l_{n}^{2}\right)$ and $\|\varphi\|_{M}<1$.

(ii) $\left|\sum_{i, j=1}^{n} \varphi(i, j)\left\langle x_{i}, y_{j}\right\rangle\right|<\left(\sum_{i=1}^{n}\left\|x_{i}\right\|^{2}\right)^{1 / 2}\left(\sum_{j=1}^{n}\left\|y_{j}\right\|^{2}\right)^{1 / 2}$

for all pairs of sets $\left\{x_{1}, \ldots, x_{n}\right\}$ and $\left\{y_{1}, \ldots, y_{n}\right\}$ of orthogonal elements in $H$.

One can show the following from Theorem 2.1.

Corollary 2.1. (i) $l^{1} \otimes l^{1} \subseteq M\left(l^{2} \otimes l^{2}\right)$ and $\|\varphi\|_{M}<K_{G}\|\varphi\|_{l^{1} \dot{\otimes} l^{1}}$, where $K_{G}$ is the Grothendieck constant.

(ii) $L\left(l^{2}\right)=\left(l^{2} \hat{\otimes} l^{2}\right)^{*} \subseteq M\left(l^{2} \hat{\otimes} l^{2}\right)$ and $\|\varphi\|_{M}<\|\varphi\|_{L(H)}$.

Now, let $\alpha, \beta: N \rightarrow N$ be any two surjective maps, not necessarily (1-1). Set $\psi=\varphi \circ \alpha \otimes \beta$, and $P^{\prime}(k)=P\left\{\alpha^{-1}(k)\right\}, Q^{\prime}(l)=Q\left(\beta^{-1}(l)\right)$. Then $P^{\prime}$ and $Q^{\prime}$ are spectral projections on $N$, whenever $P$ and $Q$ are.

A consequence of Theorem 2.1 is the following.

LEMMA 2.1. If $\varphi \in M\left(l^{2} \hat{\otimes} l^{2}\right)$, then $\varphi \circ \alpha \otimes \beta=\psi \in M\left(l^{2} \hat{\otimes} l^{2}\right)$. Further, $\|\varphi\|_{M}=\|\psi\|_{M}$.

Proof. Let $\varphi \in M\left(l^{2} \hat{\otimes} l^{2}\right)$ and $\|\varphi\|_{M}<1$. Then

$$
\begin{aligned}
\left\|\sum_{i, j=1}^{n} \psi(i, j) P(i) Q(j)\right\|_{L(H)} & =\left\|\sum_{k, l} \varphi(k, l) P^{\prime}(k) Q^{\prime}(k)\right\|_{L(H)} \\
& <\|\varphi\|_{M},
\end{aligned}
$$

by Theorem 2.1. Hence $\|\psi\|_{M}<\|\varphi\|_{M}$. Similarly, since $\alpha \otimes \beta: N \times N \rightarrow N \times N$ is onto, one can show that $\|\varphi\|_{M}<\|\psi\|_{M}$.

Lemma 2.1 implies that if $\varphi$ is a matrix in $M\left(l^{2} \hat{\otimes} l^{2}\right)$, and $\psi$ is another matrix obtained from $\varphi$ by repeating finitely many rows finitely many times then $\psi \in$ $M\left(l^{2} \hat{\otimes} l^{2}\right)$ and $\|\psi\|_{M}=\|\varphi\|_{M}$.

Let $E$ be any finite set and $\mu$ a discrete measure on $E$ which vanishes at no point of $E$. We write $\nu$ for the counting measure on $E$. If $S$ is the kernel of an operator in the unit ball of $L\left(L^{2}(E, \mu)\right)$, then $\tilde{S}(a, b)=S(a, b)(\mu(a) \mu(b))^{1 / 2}$ is the kernel of an operator in the unit ball of $L\left(L^{2}(E, \nu)\right)$. Similarly if $S$ is the kernel of an operator in 
the unit ball of $L\left(L^{2}(E, \nu)\right)$, then $\tilde{\tilde{S}}(a, b)=S(a, b) \cdot(\mu(a) \mu(b))^{-1 / 2}$ is the kernel of an operator in the unit ball of $L\left(L^{2}(E, \mu)\right)$. Now if $\varphi$ is in the unit ball of $M\left(L^{2}(E, \mu) \hat{\otimes} L^{2}(E, \mu)\right)$, then

$$
\left|\sum_{a, b \in E} \varphi(a, b) u(a) v(b) S(a, b) \mu(a) \mu(b)\right|<1
$$

for all atoms $u \otimes v$ in the unit ball of $L^{2}(E, \mu) \hat{\otimes} L^{2}(E, \mu)$ and all kernels $S$ in the unit ball of $L\left(L^{2}(E, \mu)\right)$. Then one can show that $\varphi \in M\left(L^{2}(E, \nu) \hat{\otimes} L^{2}(E, \nu)\right)$ and $\|\varphi\|_{M}<1$. Consequently we have the following.

THEOREM 2.2. Let $E$ be a finite set. Let $\nu_{1}$ and $\nu_{2}$ be any two discrete measures on $E$ which are absolutely continuous with respect to each other. Then the following are equivalent.

(i) $\varphi \in M\left(L^{2}\left(E, \nu_{1}\right) \hat{\otimes} L^{2}\left(E, \nu_{1}\right)\right)$ and $\|\varphi\|_{M}<1$.

(ii) $\varphi \in M\left(L^{2}\left(E, \nu_{2}\right) \hat{\otimes} L^{2}\left(E, \nu_{2}\right)\right)$ and $\|\varphi\|_{M}<1$.

Let $E$ be a finite set and $\nu$ a measure on $E$ with atoms $E_{1}, \ldots, E_{n}$. Set $\tilde{E}=\left\{a_{1}, \ldots, a_{n}\right\}$ with $a_{i} \in E_{i}$ and let $\tilde{\nu}$ be the measure on $\tilde{E}$ defined by $\tilde{\nu}\left(a_{i}\right)=$ $\nu\left(E_{i}\right)$. Clearly, if $\tilde{\varphi}\left(a_{i}, a_{j}\right)=\varphi\left(E_{i}, E_{j}\right)$, then $\varphi \in M\left(L^{2}(E, \nu) \hat{\otimes} L^{2}(E, \nu)\right)$ and $\|\varphi\|_{M}$ $<1$ if and only if $\tilde{\varphi} \in M\left(L^{2}(\tilde{E}, \tilde{\nu}) \hat{\otimes} L^{2}(\tilde{E}, \tilde{\nu})\right)$ and $\|\tilde{\varphi}\|_{M}<1$. Together with Theorem 2.1 and Theorem 2.2 the previous remark implies

THEOREM 2.3. The following are equivalent.

(i) $\varphi \in M\left(L^{2}(E, \nu) \hat{\otimes} L^{2}(E, \nu)\right)$ and $\|\varphi\|_{M}<1$.

(ii) $\left\|\iint_{E \times E} \varphi(a, b) d P(a) d Q(b)\right\|_{L(H)}<1$ for every pair of spectral measures $P$ and $Q$ on $(E, \nu)$.

Now, let $X$ be another set which has the same cardinality as $E$. Define $\mu$ to be a discrete measure on $X$ such that $F:(X, \mu) \rightarrow(E, \nu)$ is a (1-1) onto measure-preserving map. $F$ induces an isometric operator

$$
U: L^{2}(E, \nu) \hat{\otimes} L^{2}(E, \nu) \rightarrow L^{2}(X, \mu) \hat{\otimes} L^{2}(X, \mu),
$$

where $U(\varphi)=\varphi \circ F \otimes F$. Then we prove

THEOREM 2.4. The following are equivalent.

(i) $\varphi \in M\left(L^{2}(E, \nu) \hat{\otimes} L^{2}(E, \nu)\right)$ and $\|\varphi\|_{M}<1$.

(ii) $U(\varphi) \in M\left(L^{2}(X, \mu) \hat{\otimes} L^{2}(X, \mu)\right)$ and $\|U(\varphi)\|_{M}<1$.

Proof. (i) $\rightarrow$ (ii). By Theorem 2.2 we can assume without loss of generality that $\nu\left(E_{i}\right)=\left|E_{i}\right|$, the cardinality of $E_{i}$. Hence we take $\mu$ to be the counting measure on $X$. Theorem 2.3 together with (i) implies that $\left\|\iint_{E \times E} \varphi(a, b) d P(a) d Q(b)\right\|_{L(H)}<1$, for every pair of spectral measure $P$ and $Q$ on $(E, \nu)$.

Let $u \otimes v$ be any atom in the unit ball of $L^{2}(X, \mu) \hat{\otimes} L^{2}(X, \mu)$, and let $S$ be any unitary operator on $L^{2}(X, \mu)$. Choose two orthonormal sets of vectors in $H,\left(e_{x}\right)_{x \in X}$ and $\left(f_{y}\right)_{y \in X}$, such that $S(x, y)=\left\langle e_{x}, f_{y}\right\rangle$. Set

$$
\begin{aligned}
& P\left(E_{i}\right)=\tilde{P}\left(a_{i}\right)=\text { projection onto the span of }\left\{e_{x} \mid F(x) \in E_{i}\right\}, \\
& Q\left(E_{j}\right)=\tilde{Q}\left(a_{j}\right)=\text { projection onto the span of }\left\{f_{y} \mid F(y) \in E_{j}\right\}
\end{aligned}
$$


Thus $\left\|\Sigma_{i, j=1}^{n} \tilde{\varphi}\left(a_{i}, a_{j}\right) \tilde{P}\left(a_{i}\right) \tilde{Q}\left(a_{j}\right)\right\|_{L(H)}<1$. Further put $e=\Sigma_{x \in X} v(x) e_{x}$ and $f=$ $\Sigma_{y \in X} \bar{u}(y) f_{y}$. Then

$$
\begin{aligned}
\left|\sum_{x, y} U(\varphi)(x, y) u(x) v(y) S(y, x)\right| & =\left|\sum \tilde{\varphi}\left(a_{i}, a_{j}\right)\left\langle\sum_{F(x) \in E_{i}} u(x) e_{x}, \sum_{F(y) \in E_{j}} v(y) f_{y}\right\rangle\right| \\
& =\left|\sum \tilde{\varphi}\left(a_{i}, a_{j}\right)\left\langle\tilde{P}\left(a_{i}\right) e, \tilde{Q}\left(a_{j}\right) f\right\rangle\right|<1 .
\end{aligned}
$$

This proves (ii).

The proof of (ii) $\rightarrow$ (i) follows from the fact that $U$ is an isometry, and $U(\varphi \cdot \psi)$ $=U(\varphi) \cdot U(\psi)$. This completes the proof of the theorem.

3. Multipliers on infinite compact spaces. Let $I$ denote the unit interval $[0,1]$, and $\mathscr{F}$ be the $\sigma$-algebra of all Borel sets in $I$. For each positive integer $n$, let $\mathscr{F}_{n}$ denote the $\sigma$-algebra generated by the $2^{n}$ equal length intervals of $I$ whose union is $I$. If $\varphi$ is a bounded Borel function on $I \times I$, then $\varepsilon_{n}(\varphi)$ will denote the conditional expectation of $\varphi$ with respect to the $\sigma$-algebra $\mathscr{F}_{n} \otimes \mathscr{F}_{n}$ in $I \times I$. If $\varepsilon_{n}(\nu)$ is the restriction of $\nu$ to $\mathscr{F}_{n}$, then

$$
\iint_{E} \varphi(x, y) d \nu(x) d \nu(y)=\iint_{E} \varepsilon_{n}(\varphi)(x, y) d \varepsilon_{n}(\nu)(x) d \varepsilon_{n}(\nu)(y),
$$

for every set $E \in \mathscr{F}_{n} \otimes \mathscr{F}_{n}$. We write $L^{2}(\nu)$ for $L^{2}(I, \nu)$, and $\|F\|_{T r}$ for the norm of $F$ in $L^{2}(\nu) \hat{\otimes} L^{2}(\nu)$. Since $L^{2}(\nu) \hat{\otimes} L^{2}(\nu)$ is the dual of $L^{2}(\nu) \ddot{\otimes} L^{2}(\nu)$, [6], it follows that $\|F\|_{r r}=\sup |\langle F, \psi\rangle|$, where the supremum is taken over all compact operators $\psi$ in the unit ball of $L^{2}(\nu) \ddot{\otimes} L^{2}(\nu)$.

THEOREM 3.1. Let $\varphi, \nu, \mathscr{F}, \varepsilon_{n}(\varphi), \varepsilon_{n}(\nu)$ and $\mathscr{F}_{n}$ be as given above. Then the following are equivalent.

(i) $\varphi \in M\left(L^{2}(\nu) \hat{\otimes} L^{2}(\nu)\right)$ and $\|\varphi\|_{M}<1$.

(ii) $\varepsilon_{n}(\varphi) \in M\left(L^{2}\left(I, \mathscr{F}_{n}, \varepsilon_{n}(\nu)\right) \hat{\otimes} L^{2}\left(I, \mathscr{F}_{n}, \varepsilon_{n}(\nu)\right)\right)$ and $\left\|\varepsilon_{n}(\varphi)\right\|_{M}<1$.

Proof. (i) $\rightarrow$ (ii). For a start, let $L^{2}\left(\varepsilon_{n}(\nu)\right) \hat{\otimes} L^{2}\left(\varepsilon_{n}(\nu)\right)$ stand for $L^{2}\left(I, \mathscr{F}_{n}, \varepsilon_{n}(\nu)\right) \hat{\otimes}$ $L^{2}\left(I, \mathscr{F}_{n}, \varepsilon_{n}(\nu)\right)$. Let $f \otimes g$ be an atom in the unit ball of $L^{2}\left(\varepsilon_{n}(\nu)\right) \hat{\otimes} L^{2}\left(\varepsilon_{n}(\nu)\right)$ and $K$ be the kernel of a contractive compact operator on $L^{2}\left(\varepsilon_{n}(\nu)\right)$. Since $f \otimes g \cdot K \in$ $L^{2}\left(\varepsilon_{n}(\nu) \times \varepsilon_{n}(\nu)\right)$, it follows, [3], that

$$
\varepsilon_{n}(\varphi \cdot f \otimes g \cdot K)=f \otimes g \cdot K \cdot \varepsilon_{n}(\varphi) .
$$

This implies

$$
\left\langle\varepsilon_{n}(\varphi) \cdot f \otimes g, K\right\rangle|=|\langle\varphi \cdot f \otimes g, K\rangle \mid\langle 1 .
$$

This proves (ii).

Conversely (ii) $\rightarrow$ (i). If $K$ is the kernel of a contractive compact operator on $L^{2}(\nu)$, then $\varepsilon_{n}(K)$ is the kernel of a contractive compact operator on $L^{2}\left(\varepsilon_{n}(\nu)\right)$. Let $f \otimes g$ be an atom in the unit ball of $L^{2}(\nu) \hat{\otimes} L^{2}(\nu)$. Then by [3], $\varepsilon_{n}(f \otimes g)$ is an atom in the unit ball of $L^{2}\left(\varepsilon_{n}(\nu)\right) \hat{\otimes} L^{2}\left(\varepsilon_{n}(\nu)\right)$. The assumption (ii) implies

$$
\left|\iint_{I \times I}\left[\varepsilon_{n}(\varphi) \varepsilon_{n}(f \otimes g) \cdot \varepsilon_{n}(K)\right](x, y) d \varepsilon_{n}(\nu)(x) d \varepsilon_{n}(\nu)(y)\right|<1 .
$$


Since $\varepsilon_{n}(\varphi) \cdot \varepsilon_{n}(f \otimes g) \cdot \varepsilon_{n}(K) \rightarrow \varphi \cdot f \otimes g \cdot K$ a.e. $\nu \times \nu$, we have

$$
\begin{aligned}
& \left|\iint_{I \times I} \varphi(x, y) f(x) g(y) K(x, y) d \nu(x) d \nu(y)\right| \\
& \quad=\lim _{n}\left|\iint_{I \times I}\left[\varepsilon_{n}(\varphi) \varepsilon_{n}(f \otimes g) \varepsilon_{n}(K)\right](x, y) d \varepsilon_{n}(\nu)(x) d \varepsilon_{n}(\nu)(y)\right|<1 .
\end{aligned}
$$

This completes the proof.

Let $B(I)$ be the space of all bounded Borel functions on $I$. $\tilde{B}(I \times I, \nu \times \nu)$ denotes the space of all bounded Borel functions $\varphi$ on $I \times I$ such that there exists a bounded sequence of functions in $B(I) \hat{\otimes} B(I)$ which converges to $\varphi$ a.e. $\nu \times \nu$. We then prove

THEOREM 3.2. For a bounded Borel function $\varphi$ on $I \times I$, the following are equivalent.

(i) $\varphi \in M\left(L^{2}(\nu) \hat{\otimes} L^{2}(\nu)\right)$.

(ii) $\varphi \in \tilde{B}(I \times I, \nu \times \nu)$.

Proof. (i) $\rightarrow$ (ii). Let $\varphi \in M\left(L^{2}(\nu) \hat{\otimes} L^{2}(\nu)\right)$ and $\|\varphi\|_{M}<1$. Theorem 3.1 implies that $\varepsilon_{n}(\varphi) \in M\left(L^{2}\left(\varepsilon_{n}(\nu)\right) \hat{\otimes} L^{2}\left(\varepsilon_{n}(\nu)\right)\right)$ and $\left\|\varepsilon_{n}(\varphi)\right\|_{M}<1$. By Theorem 2.2, $\varepsilon_{n}(\varphi) \in$ $M\left(L^{2}\left(I, \mathscr{F}_{n}, \mu\right) \hat{\otimes} L^{2}\left(I, \mathscr{F}_{n}, \mu\right)\right)$, and $\left\|\varepsilon_{n}(\varphi)\right\|_{M} \leqslant 1$, where $\mu$ is the counting measure on $\mathscr{F}_{n}$. Since $M\left(l_{n}^{2} \hat{\otimes} l_{n}^{2}\right)=l_{n}^{\infty} \hat{\otimes} l_{n}^{\infty}$ (up to norm equivalence uniformly in $n$ ) [5], we get $\varepsilon_{n}(\varphi) \in B(I) \hat{\otimes} B(I)$. But $\varepsilon_{n}(\varphi) \rightarrow \varphi$ a.e. $\nu \times \nu$, it follows that $\varphi \in$ $\tilde{B}(I \times I, \nu \times \nu)$.

Conversely (ii) $\rightarrow$ (i). Let $\left(\varphi_{i}\right)_{i=1}^{\infty}$ be a sequence of functions in $B(I) \hat{\otimes} B(I)$ such that $\left\|\varphi_{i}\right\|_{M}<1$ and $\varphi_{i} \rightarrow \varphi$ a.e. $\nu \times \nu$. Theorem 3.1 implies that $\varepsilon_{n}\left(\varphi_{i}\right) \in$ $M\left(L^{2}\left(\varepsilon_{n}(\nu)\right) \hat{\otimes} L^{2}\left(\varepsilon_{n}(\nu)\right)\right)$ and $\left\|\varepsilon_{n}\left(\varphi_{i}\right)\right\|_{M}<1$, for all $i>1$. Theorem 2.2 together with the fact that $M\left(l_{n}^{2} \hat{\otimes} l_{n}^{2}\right)=l_{n}^{\infty} \hat{\otimes} l_{n}^{\infty}[5]$ implies that $\varepsilon_{n}\left(\varphi_{i}\right) \in B(I) \hat{\otimes} B(I)$. Hence $\varepsilon_{n}(\varphi) \in M\left(L^{2}\left(\varepsilon_{n}(\nu)\right) \hat{\otimes} L^{2}\left(\varepsilon_{n}(\nu)\right)\right)$ and $\left\|\varepsilon_{n}(\varphi)\right\|_{M}<1$. By Theorem 3.1, $\varphi \in$ $M\left(L^{2}(\nu) \hat{\otimes} L^{2}(\nu)\right)$ and $\|\varphi\|_{M}<1$. This completes the proof.

In the final part of the paper we prove a result similar to Theorem 2.3. To start with, let $P$ and $Q$ be any two spectral measures on $(I, \mathcal{F})$, and $\varepsilon_{n}(P)$ and $\varepsilon_{n}(Q)$ will denote the restriction of $P$ and $Q$ to $\left(I, \mathscr{F}_{n}\right)$ respectively. If $\varphi$ is a bounded Borel function on $(I \times I, \mathscr{F} \times \mathscr{F})$, then set

$$
S_{n}=\iint_{I \times I} \varepsilon_{n}(\varphi)(x, y) d \varepsilon_{n}(P)(x) d \varepsilon_{n}(Q)(y) .
$$

The sequence $\left(S_{n}\right)_{n=1}^{\infty}$ need not converge in the weak-operator topology. However if $\left(S_{n}\right)_{n=1}^{\infty}$ converges weakly, then let $S=\iint_{I \times I} \varphi(x, y) d P(x) d Q(y)$ denote such a limit. One observes that if $S$ exists then it is independent of the sequence $\left(\mathscr{F}_{n}\right)_{n=1}^{\infty}$. Now we prove

THEOREM 3.3. For any bounded Borel function $\varphi$ on $I \times I$ and for any Borel measure $\nu$ on $I$, the following are equivalent.

(i) $\varphi \in M\left(L^{2}(\nu) \hat{\otimes} L^{2}(\nu)\right)$ and $\|\varphi\|_{M} \leqslant 1$.

(ii) $\left\|\iint_{I \times I} \varphi(x, y) d P(x) d Q(y)\right\|_{L(H)} \leqslant 1$ for all pairs of spectral measures $P$ and $Q$ on $(I, \mathscr{F})$. 
Proof. (i) $\rightarrow$ (ii). Let $\varphi \in M\left(L^{2}(\nu) \hat{\otimes} L^{2}(\nu)\right)$ and $\|\varphi\|_{M}<1$. Theorem 3.1 implies that $\varepsilon_{n}(\varphi) \in M\left(L^{2}\left(\varepsilon_{n}(\nu)\right) \hat{\otimes} L^{2}\left(\varepsilon_{n}(\nu)\right)\right)$ and $\left\|\varepsilon_{n}(\varphi)\right\|_{M}<1$ for all $n>1$. Hence by Theorem 2.3, $\left\|S_{n}\right\|_{L(H)}<1$ for all $n>1$. Now we want to prove that $\left(\left\langle S_{n} a, b\right\rangle\right\rangle_{n-1}^{\infty}$ is a convergent sequence of complex numbers for every $a$ and $b$ in $H$. We show such a sequence is a Cauchy sequence.

If $E=A \times B$ is an atom in $\mathscr{F}_{n} \times \mathscr{F}_{n}$, then for $m>n, E \in \mathscr{F}_{m} \times \mathscr{F}_{m}$. Let $E=\sum_{i, j=1}^{k} A_{i} \times B_{j}$ in $F_{m} \times F_{m}$, where $A=\cup A_{i}, B=\cup B_{j}$ and $A_{i} \times B_{j}$ is an atom in $\mathscr{F}_{m} \times \mathscr{F}_{m}$. If $\varepsilon_{n}(\varphi)(E)=\lambda \in \mathbf{C}$, then

$$
\begin{aligned}
\varepsilon_{n}(\varphi)(E)\langle P(A) a, Q(B) b\rangle & =\lambda\langle P(A) a, Q(B) b\rangle \\
& =\sum_{i, j=1}^{n} \lambda\left\langle P\left(A_{i}\right) a, Q\left(B_{j}\right) b\right\rangle .
\end{aligned}
$$

Hence for each $m>n$, we have a function $\varepsilon_{n}^{m}(\varphi)$ which is $\mathscr{F}_{m} \times \mathscr{F}_{m}$ measurable and $\varepsilon_{n}^{m}(\varphi)(x, y)=\varepsilon_{n}(\varphi)(x, y)$ for all $(x, y) \in I \times I$. This enables us to write

$$
\left|\left\langle S_{n} a, b\right\rangle-\left\langle S_{m} a, b\right\rangle\right|=\left|\sum_{r, s}\left(\varepsilon_{n}^{m}(\varphi)(r, s)-\varepsilon_{m}(\varphi)(r, s)\right) \cdot\left\langle Q\left(E_{s}\right) a, P\left(E_{r}\right) b\right\rangle\right|
$$

where $\left(E_{r} \times E_{s}\right)_{r, s=1}^{2^{m}}$ are the atoms in $\mathscr{F}_{m} \times \mathscr{F}_{m}$. To estimate the last summation, we estimate $\left\|\varepsilon_{n}^{m}(\varphi)-\varepsilon_{m}(\varphi)\right\|_{M}$ as an element of $M\left(L^{2}\left(\varepsilon_{m}(\nu)\right) \hat{\otimes} L^{2}\left(\varepsilon_{m}(\nu)\right)\right)$.

Let $u \otimes v$ be an atom in the unit ball of $L^{2}\left(\varepsilon_{m}(\nu)\right) \hat{\otimes} L^{2}\left(\varepsilon_{m}(\nu)\right)$ and $K$ a contractive compact operator on $L^{2}\left(\varepsilon_{m}(\nu)\right)$. Since $\varepsilon_{m}(\varphi \cdot u \otimes v \cdot K)=\varepsilon_{m}(\varphi) \cdot u \otimes v$ $\cdot K$, and $\varepsilon_{n}^{m}(\varphi) \stackrel{n, m}{\rightarrow} \varphi$ a.e. $\nu \times \nu$, we get

$$
\left\langle\left(\varepsilon_{n}^{m}(\varphi)-\varepsilon_{m}(\varphi)\right) u \otimes v, K\right\rangle \stackrel{n, m}{\rightarrow} 0 .
$$

Theorem 2.3 now implies that

$$
\lim _{n, m}\left|\left\langle S_{n} a, b\right\rangle-\left\langle S_{m} a, b\right\rangle\right|=0 .
$$

This proves (ii).

Conversely (ii) $\rightarrow$ (i). The assumption implies that for each $\delta>0$ there exists an $r(\delta)$ such that $\left\|S_{r}\right\|_{L(H)}<1+\delta$. Choose a sequence $\delta_{r} \rightarrow 0$ as $r \rightarrow \infty$ and $\left\|S_{r}\right\|_{L(H)}$ $<1+\delta_{r}$. By Theorem 2.3 we have

$$
\varepsilon_{r}(\varphi) \in M\left(L^{2}\left(\varepsilon_{r}(\nu)\right) \hat{\otimes} L^{2}\left(\varepsilon_{r}(\nu)\right)\right) \text { and }\left\|\varepsilon_{r}(\varphi)\right\|_{M}<1+\delta_{r} .
$$

For all atoms $u \otimes v$ of unit norm in $L^{2}(\nu) \hat{\otimes} L^{2}(\nu)$ and all contractive compact operators $K$ on $L^{2}(\nu)$ we have

$$
\begin{aligned}
|\langle\varphi \cdot u \otimes v, K\rangle| & =\lim _{r}\left|\left\langle\varepsilon_{r}(\varphi) \cdot \varepsilon_{r}(u \otimes v), \varepsilon_{r}(K)\right\rangle\right| \\
& <\lim _{r}\left(1+\delta_{r}\right)<1 .
\end{aligned}
$$

This completes the proof of the theorem.

\section{BiBLIOGRAPHY}

1. I. Amemiya and K. Shiga, On tensor product of Banach spaces, Kōdai Math. Sem. Rep. 9 (1957), 161-176.

2. G. Bennett, Schur multipliers, Duke Math. J. 44 (1977), 613-639.

3. P. Billingsley, Ergodic theory and information, Wiley, New York, 1965.

4. J. Diestel and J. R. Uhl, Vector measures, Math. Surveys, no. 15, Amer. Math. Soc., Providence, R. I., 1977. 
5. R. Khalil, Ph.D. thesis, McGill University, 1978.

6. J. R. Ringrose, Compact non-self adjoint operators, Van Nostrand Reinhold, London, 1971.

7. R. Schatten, Norm ideals of completely continuous operators, Springer-Verlag, Berlin and New York, 1960.

8. J. Schur, Bemerkungen zur Theorie der beschränkten Bilinearformen mit unendlich vielen Veränderlichen, J. Reine Angew. Math. 140 (1911), 1-28.

Department of Mathematics, MCGil. Universtry, 805 Shiberooke St. W., Montran, P. Q., Canada H3A $2 \mathrm{~K} 6$

Current address: Department of Mathematics, Kuwait University, Kuwait 